\title{
The Role of Cervical Mediastinoscopy in Determining N Status and Morphological Verification of Lung Cancer
}

\author{
Roman Vereshchako ${ }^{1}$, Ihor Sukhin ${ }^{1,2}$, Oleksandr Piskorskyi ${ }^{1,2}$, Roman Gylevych ${ }^{2}$, Yehor Tereshchenko ${ }^{1}$ \\ ${ }^{\mathrm{T}} \mathrm{MD}, \mathrm{PhD}$, Professor, Doctor of Medical Sciences, Bogomolets National Medical University, Kyiv, Ukraine \\ ${ }^{2} \mathrm{MD}, \mathrm{PhD}$, Associate Professor, Doctor of Medical Sciences, Kyiv Railway Clinical Hospital N3, Kyiv, Ukraine \\ *Corresponding Author \\ Roman Vereshchako
}

\section{Article History}

Received: 30.04 .2020

Accepted: 11.05 .2020

Published: 16.05 .2020

\begin{abstract}
Lung cancer remains the leading cause of cancer mortality and is ranked first on the cancer prevalence against planet's population. The role of cervical mediastinoscopy in determining $\mathrm{N}$ status and morphological verification of lung cancer is considered in the article. Material and research methods: The study included 85 patients aged from 19 to 76 years (mean age was 53 \pm 13.4 years). Men and women were $47(55.3 \%)$ and $38(44.7 \%)$ respectively. General clinical, laboratory, endoscopic, chest radiography, morphological, immunohistochemical, and statistical methods were used. An indication for mediastinoscopy was the enlargement of the lymph nodes of the mediastinum revealed by thorax computed tomography. Results: The frequency of different combinations in the studied groups of nodes was presented as follows: 2R, 4R, $7-16,38 \%$; 4R, 4L, $7-21.1 \%$; 4L, $7-9,72 \%$; 2R, 4R - 7,73\%; 4R, 4L - 6,2\%; 4R, $7-9,02 \%$; 4R $2,0 \% ; 10 \mathrm{~L}, 4 \mathrm{~L}, 7-2,72 \% ; 2 \mathrm{R}, 7-1,83 \% ; 2 \mathrm{R}, 2 \mathrm{~L}, 4 \mathrm{R}, 4 \mathrm{~L}-4,9 \% ; 2 \mathrm{~L}, 4 \mathrm{~L}, 10 \mathrm{~L}, 4 \mathrm{R}-1,16 \% ; 7-2,8 \% ; 2 \mathrm{R}, 4 \mathrm{R}, 7-$ $7,55 \% ; 2 \mathrm{R}, 4 \mathrm{R}, 4 \mathrm{~L}, 7-5,34 \%, 4 \mathrm{R}, 10 \mathrm{R}-1,55 \%$. At 85 patients with enlarged mediastinal lymph nodes on tomography, lung cancer was verified in 42 patients. 43 patients presented with another oncological or non-oncological pathology. In 42 patients, a study of lymph nodes of the mediastinum allowed to verify the diagnosis of lung cancer 25 patients and determine the status of $\mathrm{N} 17$ patients. The sensitivity of cervical mediastinoscopy, compared with computed tomography (CT), was $97.6 \mathrm{CI} \% 95$ - [89.3-100.0]. According to chest CT in patients with lung cancer, an increase in mediastinal lymph nodes from 10 to $14.9 \mathrm{~mm}$ was observed in $47(55.3 \%)$ people and more than $15 \mathrm{~mm}$ in 38 (44.7\%) people. The presence of lung cancer metastases in the mediastinal nodes was detected by mediastiniscopy in 33 (78.57\%) patients. In $9(21.43 \%)$ cases, mediastinal lymphadenopathy (according to CT data) was not caused by metastasis (false positive). The histological distribution after cervical mediastinoscopy was as follows: squamous cell carcinoma - $10(21.43 \%)$, adenocarcinoma - 24 (57.1\%), small cell carcinoma - 8 (19.04\%), observations respectively. The results of postoperative histological examination of the mediastinal lymph nodes coincided with the histological results of preoperative cervical mediastinoscopy in $100 \%$ of cases (100.0\% CI [97.1-100.0]). Conclusions: Cervical mediastinoscopy in patients with primary lung cancer made it possible precisely determine the cause of enlargement of mediastinal lymph nodes and to set the frequency of different combinations of the studied groups of nodes was presented as follows. In patients with primary lung cancer, this method verify the diagnosis in $40.5 \%$ of cases and determine $\mathrm{N}$ status in $100 \%$ of cases. The sensitivity of the cervical mediastinoscopy method, compared with computed tomography, was 96.4\% CI [90.8-99.3]. The results of the postoperative histological examination of the mediastinal lymph nodes coincided with the histological results of preoperative cervical mediastinoscopy in $100 \%$ of cases.
\end{abstract}

Keywords: lung cancer, cervical mediastinoscopy, diagnostic value, sensitivity, specificity.

\section{Actuality}

Lung cancer remains the leading cause of cancer mortality and is ranked first from cancer prevalence in the world's population $[8,9]$. According to the data by the European Association of Oncology, there are approximately 1.6 million new cases of lung cancer annually worldwide [13]. Men are more likely to suffer from women - 33.8 per 100,000 versus 13.5 per 100,000 population [7]. Early diagnosis is essential to overcome this pathology, allowing early and

Copyright @ 2020: This is an open-access article distributed under the terms of the Creative Commons Attribution license which permits unrestricted use, distribution, and reproduction in any medium for non commercial use (NonCommercial, or CC-BY-NC) provided the original author and source are credited. 
personalized treatment to begin [11]. The main task of lung cancer diagnostics is to determine the location, size, propagation and morphological verification of the tumor.

Non-invasive and invasive diagnostic methods are used to diagnose of lung cancer (LC). The main non-invasive methods include: laboratory examinations, chest radiography, magnetic resonance imaging (MRI), computed tomography (CT), positron emission computed tomography (PET/CT), osteoscintigraphy, which can be set the presence, spread and localization of oncological growth. These methods are applied as a first stage of diagnostic search and allow determining the invasive method, which is carried out for the target morphological verification $[12,14,22]$.

Later manifestation of the disease very often leads to involvement of the lymph nodes at the time of diagnosis and can be a reason of a limitation of the surgical component of the treatment of the disease. On the one hand, indications for the use of invasive diagnostic methods to confirm mediastinal lymph node involvement are currently well defined and well understood. These are, in particular, enlarged lymph nodes of the mediastinum detected in computed tomography, central localized tumors, enlarged N1 lymph nodes without enlargement of the mediastinal ones [4].

Histological diagnosis is the main reason to establish the involving of malignancy by the lymph nodes, as it depends on the prognosis and the choice of adequate treatment. X-ray signs often do not correlate with the morphological manifestations of the disease, making it difficult to choose the right therapeutic decision. There are various techniques for obtaining a sample of the lymph node of the mediastinum.

In practice, the situation is quite common when the patient has histologically confirmed lung cancer, and there is no morphological confirmation of lesions of the intrathoracic lymph nodes. The question arises as to the benefit of using invasive research methods in massive mediastinal infiltration confirmed by other methods [10].

For invasive diagnosis of lung cancer, we use transthoracic puncture biopsy, fibrobronchoscopy with biopsy, transbronchial biopsy (EBUS TBNA), transesophageal biopsy (EUS-FNA), videotoracoscopy with biopsy, cervical mediastinoscopy (CM) and open biopsy [15, 20, 23].

In 1959, E. Carlens was the first who described mediastinoscopy, a study of the upper anterior mediastinum using a specific tool he developed, a mediastinoscope. The main indication for this surgical intervention at that time was to assess the condition of the mediastinal lymph nodes to clarify the stage of lung cancer. Cervical mediastinoscopy by E. Carlens allows to evaluate the condition of the anterior-superior mediastinum of the trachea, major bronchi, upper and lower paratracheal lymph nodes, right and left, bifurcation lymph nodes [5]. It can be used in case of suspected lymphogenous metastasis of verified lung cancer in order to clarify the degree of prevalence of the process, in cases of unverified lung cancer, to clarify the diagnosis and to determine the stage, to diagnose isolated mediastinal or intrathoracic lymphadenopathy [2].

The main advantage of this method is its high diagnostic value - sensitivity is set at $81.8 \%$, and specificity $100 \%$ [19]. This method allows the sampling of morphological material in sufficient quantity to perform histological, immunohistochemical and molecular investigation, which makes it possible to objectively diagnose and reasonably prescribe treatment.

Histological evaluation of mediastinal lymph nodes is possible when using invasive diagnostic methods such as VATS, EBUS-TBNA, cervical mediastinoscopy [17, 21]. The disadvantages of VATS can be considered the invasiveness of the technique, the ability to perform only unilateral biopsy of lymph nodes, long admission in hospital [1, 6]. The disadvantages of EBUS-TBNA are expensive equipment and the ability to perform biopsy of a limited amount of material $[16,18]$.

Staging of lung cancer is a major indication for mediastinoscopy. The main advantage of mediastinoscopy over other investigations is that this method provides morphological confirmation of tumor propagation. Thus, in a retrospective analysis of $72 \%$ of patients in whom a lesion of the intra-thoracic lymph nodes was revealed by mediastinoscopy, chest radiography was uninformative. Chest CT has expanded its diagnostic capabilities, however, research suggests that chest CT cannot and should not be used as a stand-alone method for lung cancer staging, as the method reliably detects mediastinal lymph nodes only in 52-58\% of cases. A CT scan can detect any affected lymph nodes larger than $1 \mathrm{~cm}$ in size, but the frequency of false positives is approximately $30 \%$. On average, the sensitivity and specificity of chest CT when detecting intrathoracic lymphogenous metastases of lung cancer is equal to 56-63\% and 52$57 \%$, respectively, whereas with mediastinoscopy these indicators reach $98-100 \%$ and $95-97 \%$, respectively. In terms of sensitivity and specificity $(80 \%$ and $96-98 \%$, respectively), positron emission tomography approaches the mediastinoscopy $[4,24]$. 
As you can see, despite the presence of a large number of scientific papers, local and international recommendations, the issue of early diagnosis of this disease remains an actual and debatable problem.

In addition, in the context of the implementation of European standards for the diagnosis and treatment of lung cancer, it is necessary to choose the optimal diagnostic technique for the evaluation of mediastinal lymph nodes in order to improve the effectiveness of treatment of this complex pathology.

Therefore, the purpose of our work was to study the role of cervical mediastinoscopy in the diagnosis of lung cancer.

\section{Material ANd ResearCh Methods}

The work was performed during 2016-2020 based on the oncology clinic of the Department of Oncology of the O.O. Bogomolets National Medical University (Kiev, Ukraine) and the Kiev Clinical Railway Transport Hospital No. 3.

The study included 85 patients aged 19 to 76 years (mean age was $53 \pm 13.4$ years). The proportion of men and women were $47(55.3 \%)$ and $38(44.7 \%)$ respectively.

Between 85 patients, 68 people $(80 \%)$ had cancer: kidney cancer - 1 patient, thyroid cancer - 2, melanoma - 2 , gastric cancer - 2, breast cancer - 5, colorectal cancer - 2, lymphoma - 11, cancer prostate cancer - 1 , lung cancer - 42 . Medininal lymphadenopathy unrelated to oncology was $17(20 \%)$ patients: sarcoidosis (6), granulomatous inflammation (1), tuberculosis (3), Castelman's disease (1), nonspecific inflammation (6).

We have used general clinical, laboratory, endoscopic (EBUS-TBNA, CMM), radiographical (CT, PET-CT), morphological, immunohistochemical and statistical methods of study.

An indication for mediastinoscopy was the enlargement of the lymph nodes of the mediastinum revealed by computed tomography of the thoracic organs. Mediastinoscopy was performed under general anesthesia. Used a classic mediastinoscopy according to Carlens in the position of the patient on his back with a roller under his shoulders. The first anatomical landmark is a jugular notch, directly above which a skin incision was made in the lower third of the neck in the transverse direction. Musculus platysma, along with the sternal and sublingual muscles, moved along the white line of the neck. The second anatomical landmark is the trachea. After dissection of the pre-tracheal fascia, the trachea was exposed and an index finger was inserted into the wound canal, the paratracheal tissue was stratified, and a course was inserted for the insertion of a mediastinoscope. His blade was moved along the trachea, focusing on its rings. The third anatomical landmark is tracheal bifurcation. Paratracheal areas, the area of the tracheal bifurcation and the initial areas of the main bronchi were examined. Enlarged lymph nodes were taken for biopsy (excision biopsy), from different sites, right and left. To examine the root of the left lung, the blade of the mediastinoscope was mounted on the anterior semicircle of the left main bronchus, the dissector was stratified by peribronchial tissue and anterior from the bronchus was separated by the posterior semicircle of the left pulmonary artery; The mediastinoscope was advanced into the formed canal to the area of the pulmonary artery and the beginning of the maxillary bronchus.

Patients were discharged from the hospital the day of surgery or the next day. The study was conducted in accordance with the requirements of the Helsinki Declaration of Human Rights and all informed participants gave their written informed consent.

For statistical analysis results, we used Statistica for Windows Version 10.0 (Stat Soft inc., USA). Parameters are presented in the form $\mathrm{M} \pm \mathrm{m}$, where $\mathrm{M}$ is the mean, $\mathrm{m}$ is standard deviation. At the case of $\mathrm{p}<0.05$, differences were set as statistically significant.

\section{RESEARCH RESULTS}

The frequency of lymph nodes used for the study was as follows: $7-38$ patients, $2 \mathrm{R}-17$ patients, $2 \mathrm{~L}-6$ patients, 4R - 39 patients, 4L - 29 patients, 10R - 3 patients, 10L - 1 patients.

The frequency of various combinations of the studied groups of nodes was presented as follows: $2 \mathrm{R}, 4 \mathrm{R}, 7-$ 16,38\%; 4R, 4L, 7 - 21.1\%; 4L, 7 - 9,72\%; 2R, 4R - 7,73\%; 4R, 4L - 6,2\%; 4R, 7 - 9,02\%; 10L, 4L, 7 - 2,72\%; 2R, 7 $1,83 \%$; 2R, 2L, 4R, 4L-4,9\%; 2L, 4L, 10L, 4R - 1,16\%; $7-2,8 \% ; 2 \mathrm{R}, 4 \mathrm{R}, 7-7,55 \%$; 2R, 4R, 4L, $7-5,34 \%, 4 \mathrm{R}, 10 \mathrm{R}$ $-1,55 \%$. 
Table-1: Ratio of different combinations in affected lymph nodes at the patients with lung cancer

\begin{tabular}{|l|l|}
\hline Parameter & $\%$ \\
\hline 2R, 4R & 16,38 \\
\hline 4R, 4L & 21.1 \\
\hline 4L & 9,72 \\
\hline 2R, 4R & 7,73 \\
\hline $4 \mathrm{R}, 4 \mathrm{~L}$ & 6,2 \\
\hline 4R & 9,02 \\
\hline 10L, 4L & 2,72 \\
\hline 2R & 1,83 \\
\hline 2R, 2L, 4R, 4L & 4,9 \\
\hline 2L, 4L, 10L, 4R & 1,16 \\
\hline 2R, 4R & 7,55 \\
\hline 2R, 4R, 4L & 5,34 \\
\hline 4R,10R & 1,55 \\
\hline
\end{tabular}

The variety of combinations is due to the fact that, first of all, the enlarged lymph nodes were investigated. Most often, the samples of 3 groups of lymph nodes were taken.

The main purpose of cervical mediastinoscopy among patients with lung cancer was to determine the status of $\mathrm{N}$. In 42 patients, the study of lymph nodes of the mediastinum allowed to verify the diagnosis of lung cancer and determine the status of N. That is, the sensitivity of the method, compared with computed tomography, was $97.6 \mathrm{CI} \% 95$ [89.3-100.0]. In addition, a reliable Specificity indicator was established at 46.5 CI\%95 - [32.3-61.2] and a low probability of False negative rate - 0.05 CI\%95 - [0.01-0.26] Table-2.

Table-2: Parameters of Diagnostic Value Neck Mediastinoscopy versus Computed Tomography at the Patients with Lung Cancer

\begin{tabular}{|l|l|}
\hline PARAMETER & VALUE [CI\%95] \\
\hline Sensitivity & $97.6[89.3-100.0]$ \\
\hline Specificity & $46.5[32.3-61.2]$ \\
\hline False positive rate & $53.5[38.9-67.7]$ \\
\hline False negative rate & $2.33[0.37-10.7]$ \\
\hline Likelihood ratio for positive test & $1.83[1.44-2.31]$ \\
\hline Likelihood ratio for negative test & $0.05[0.01-0.26]$ \\
\hline Youden's index & $0.44[0.31-0.57]$ \\
\hline Area under ROC curve & $72.1[65.6-78.6]$ \\
\hline Diagnostic odds ratio & $36.5[6.4-97.8]$ \\
\hline
\end{tabular}

According to chest CT in patients with lung cancer, an increase in the mediastinal lymph nodes from 10 to 14.9 $\mathrm{mm}$ was observed in $23(54,76 \%)$, and more than $15 \mathrm{~mm}$ in $19(45,24 \%)$. The presence of lung cancer metastases in the mediastinal nodes was detected in $33(78,57 \%)$ patients. In $9(21,43 \%)$ subjects, mediastinal lymphadenopathy (according to CT data) was not caused by metastasis. The histological distribution after performing cervical mediastinoscopy was as follows: squamous cell carcinoma - $10(23,8 \%)$ cases, adenocarcinoma - $24(57.1 \%)$, small cell carcinoma - 8 (19,04\%). According to the classification, TNM - T4N3M0 - 3, T4N2M0 - 4, T4N2M1 - 2, T3N3M1 - 3, T3N2M0 - 3, T3N0M0 - 3, T2N3M1 - 2, T2N3M0 - 6, T2N2M1 - 4, T2N2M0 - 5, T2N0M1 - 1, T2N0M0 - 5, T1N2M0 - 2, Table-3.

Table-3: Ratio of different stages by TNM classification at the patients with lung cancer

\begin{tabular}{|l|l|l|}
\hline Parameter & Number of cases & $\mathbf{\%}$ \\
\hline T4N3M0 & 3 & 7,1 \\
\hline T4N2M0 & 4 & 9,5 \\
\hline T4N2M1 & 2 & 4,8 \\
\hline T3N3M1 & 3 & 7,1 \\
\hline T3N2M0 & 3 & 7,1 \\
\hline T3N0M0 & 3 & 7,1 \\
\hline T2N3M1 & 2 & 4,8 \\
\hline T2N3M0 & 6 & 14,3 \\
\hline T2N2M1 & 4 & 9,5 \\
\hline T2N2M0 & 5 & 11,9 \\
\hline T2N0M1 & 1 & 2,3 \\
\hline T2N0M0 & 5 & 11,9 \\
\hline T1N2M0 & 2 & 4,8 \\
\hline
\end{tabular}


The results of postoperative histological examination of mediastinal lymph nodes coincided with the histological results of preoperative cervical mediastinoscopy in 100\% of cases (100.0 [97.1-100.0]). False negative rate 0.01 [0.252.89] was unreliable. Performing cervical mediastinoscopy influenced the choice of treatment tactics.

\section{Conclusions}

Thus, cervical mediastinoscopy in patients with primary lung cancer allowed:

1. Accurately establish the cause of the increase in mediastinal lymph nodes and determine the frequency of various combinations of the studied groups.

2. In patients with primary lung cancer, verify the diagnosis in $100 \%$ of cases and determine the $\mathrm{N}$ status in $100 \%$ of cases.

3. The sensitivity of the method of cervical mediastinoscopy, compared with computed tomography, was 97.6\%CI[89.3-100.0]; a reliable Specificity of 46.5\%CI [32.3-61.2] and a low probability of False negative rate of the test $-0.05 \% \mathrm{CI}[0.01-0.26]$.

4. The results of postoperative histological examination of the mediastinal lymph nodes coincided with the histological results of preoperative cervical mediastinoscopy in 100\% of cases (100.0 [97.1-100.0]). False negative rate 0.01 [0.25-2.89] was unreliable.

5. Cervical mediastinoscopy made it possible to collect enough tissue samples for immunohistochemical study, to accurately establish the diagnosis and substantiate the treatment of patients with primary lung cancer.

\section{REFERENCES}

1. Abbas, A. E. (2018). Surgical management of lung cancer: History, evolution, and modern advances. Current oncology reports, 20(12), 98.

2. Avasarala, S. K., Aravena, C., \& Almeida, F. A. (2020). Convex probe endobronchial ultrasound: historical, contemporary, and cutting-edge applications. Journal of Thoracic Disease, 12(3), 1085-1099.

3. Bousema, J. E., Heineman, D. J., Dijkgraaf, M. G., Annema, J. T., \& van den Broek, F. J. (2020). Adherence to the mediastinal staging guideline and unforeseen N2 disease in patients with resectable non-small cell lung cancer: Nationwide results from the Dutch Lung Cancer Audit-Surgery. Lung Cancer, 142, 51-58.

4. Bousema, J. E., Heineman, D. J., Dijkgraaf, M. G., Annema, J. T., \& van den Broek, F. J. (2020). Adherence to the mediastinal staging guideline and unforeseen N2 disease in patients with resectable non-small cell lung cancer: Nationwide results from the Dutch Lung Cancer Audit-Surgery. Lung Cancer, 142, 51-58.

5. Calenda, E., Peillon, C., \& Baste, J. M. (2013). Double lumen tube with hook (Carlens) and tracheostomy. Acta anaesthesiologica Taiwanica: official journal of the Taiwan Society of Anesthesiologists, 51(4), 187.

6. Cheng, A. M., \& Wood, D. E. (2015). VATS versus open surgery for lung cancer resection: moving beyond the incision. Journal of the National Comprehensive Cancer Network, 13(2), 166-170.

7. de Groot, P. M., Wu, C. C., Carter, B. W., \& Munden, R. F. (2018). The epidemiology of lung cancer. Translational lung cancer research, 7(3), 220-233.

8. Ezemba, N., Ekpe, E. E., \& Eze, J. C. (2012). Challenges of lung cancer management in a developing country. Nigerian journal of medicine: journal of the National Association of Resident Doctors of Nigeria, 21(2), 214-217.

9. Hamra, G. B., Guha, N., Cohen, A., Laden, F., Raaschou-Nielsen, O., Samet, J. M., ... \& Loomis, D. (2014). Outdoor particulate matter exposure and lung cancer: a systematic review and meta-analysis. Environmental health perspectives, 122:906-911.

10. Hylton, D. A., Turner, S., Kidane, B., Spicer, J., Xie, F., Farrokhyar, F., ... \& Hanna, W. C. (2019). The Canada Lymph Node Score For Prediction of Malignancy in Mediastinal Lymph Nodes During Endobronchial Ultrasound. The Journal of Thoracic and Cardiovascular Surgery. S0022-5223(19)33477-4.

11. Jemal, A., \& Fedewa, S. A. (2017). Lung cancer screening with low-dose computed tomography in the United States-2010 to 2015. JAMA oncology, 3(9), 1278-1281.

12. Jones, G. S., \& Baldwin, D. R. (2018). Recent advances in the management of lung cancer. Clinical Medicine, 18(Suppl 2), s41.

13. Malvezzi, M., Carioli, G., Bertuccio, P., Boffetta, P., Levi, F., La Vecchia, C., \& Negri, E. (2017). European cancer mortality predictions for the year 2017, with focus on lung cancer. Annals of Oncology, 28(5), 1117-1123.

14. Nasim, F., Sabath, B. F., \& Eapen, G. A. (2019). Lung Cancer. Med Clin North Am. 103(3): 463-473.

15. Nasu, S., Okamoto, N., Suzuki, H., Shiroyama, T., Tanaka, A., Samejima, Y., ... \& Ueda, K. (2019). Comparison of the Utilities of Cryobiopsy and Forceps Biopsy for Peripheral Lung Cancer. Anticancer Research, 39(10), 56835688 .

16. Sakakibara, R., Inamura, K., Tambo, Y., Ninomiya, H., Kitazono, S., Yanagitani, N., ... \& Mun, M. (2017). EBUSTBNA as a promising method for the evaluation of tumor PD-L1 expression in lung cancer. Clinical lung cancer, 18(5), 527-534. 
17. Sehgal, I. S., Dhooria, S., Aggarwal, A. N., Behera, D., \& Agarwal, R. (2016). Endosonography versus mediastinoscopy in mediastinal staging of lung cancer: systematic review and meta-analysis. The Annals of thoracic surgery, 102(5), 1747-1755.

18. Şimon, M., Pop, B., Toma, I. L., Vallasek, A. K., \& Şimon, I. (2017). The use of EBUS-TBNA and ROSE in the diagnosis of lung cancer. Rom J Morphol Embryol, 58(1), 79-87.

19. Sivrikoz, C. M., Ak, I., Simsek, F. S., Döner, E., \& Dündar, E. (2012). Is mediastinoscopy still the gold standard to evaluate mediastinal lymph nodes in patients with non-small cell lung carcinoma?.The Thoracic and cardiovascular surgeon, 60(02), 116-121.

20. Song, Z., \& Liu, Y. (2018). Progress of liquid biopsy in early diagnosis of lung cancer. Zhongguo fei ai za zhi= Chinese journal of lung cancer, 21(8), 620-627.

21. Motta, J. P. S., e Silva, J. R. L., Lobato, C. S., Mendonça, V. S., \& Steffen, R. E. (2019). Endobronchial ultrasoundguided transbronchial needle aspiration versus mediastinoscopy for mediastinal staging of lung cancer: A protocol for a systematic review of economic evaluation studies. Medicine, 98(39).

22. Stiles, B. M., Pua, B., \& Altorki, N. K. (2016). Screening for Lung Cancer. Surgery Oncology Clinical New American. 25(3):469-479.

23. Wijmans, L., de Bruin, D. M., Meijer, S. L., \& Annema, J. T. (2016). Real-time optical biopsy of lung cancer. American journal of respiratory and critical care medicine, 194(8), e10-e11.

24. Zhang, L., Wu, F., Zhu, R., Wu, D., Ding, Y., Zhang, Z., ... \& Mediastinal N Staging Assessment Working Group. (2020). Application of computed tomography, positron emission tomography-computed tomography, magnetic resonance imaging, endobronchial ultrasound, and mediastinoscopy in the diagnosis of mediastinal lymph node staging of non-small-cell lung cancer: A protocol for a systematic review. Medicine, 99(9), e19314. 